\title{
Current therapeutic protocols for chronic granulomatous fungal sinusitis*
}

V. Rupa, S. Maheswaran, J. Ebenezer, S.S. Mathews

Department of ENT, Christian Medical College, Vellore, India
Rhinology 53: 181-186, 2015

DOl:10.4193/Rhino 14.183

*Received for publication:

July 26,2014

Accepted: December 13, 2014

\section{Abstract}

Background: The treatment of chronic granulomatous fungal sinusitis (CGFS), a rare form of invasive fungal sinusitis, is controversial.

Aim: To assess the response to postoperative antifungal therapy in patients with CGFS and suggest an effective treatment protocol.

Methodology: Clinical records of patients with CGFS who had undergone excisive surgery followed by antifungal therapy were reviewed to assess current disease status.

Results: Fourteen male and 4 female patients were diagnosed with CGFS, based on typical histopathological and fungal smear/ culture results. Aspergillus flavus was isolated from $88.9 \%$ cases. Stage 1 patients had resectable sinonasal disease, stage 2 had additional spread to orbit/palate and stage 3 had extensive disease. Follow up ranged from 6 months to 8 years. Residual disease was seen in all but one patient who received amphotericin B as first line therapy and in none of those who received itraconazole or voriconazole. Even those who received azoles as second line therapy were disease free at last follow-up.

Conclusion: Surgery followed by itraconazole or voriconazole for Stage 1 and 2 disease and voriconazole for stage 3 disease is recommended for a good outcome. Amphotericin B is not recommended as first line therapy for CGFS.

Keywords: Chronic granulomatous fungal sinusitis, amphotericin B, itraconazole, voriconazole

\section{Introduction}

Chronic granulomatous fungal sinusitis (CGFS) is a unique, chronic form of invasive fungal sinusitis seen in immunocompetent patients, typically from Sudan, India, Pakistan and Saudi Arabia. The disease, which runs an indolent course, chiefly involves the maxillary and ethmoid sinuses, nose, orbit and cheek ${ }^{(1,2)}$. The distinctive presence of non-caseating granuloma containing fungal hyphae differentiates this disease from chronic invasive fungal sinusitis, a disease more commonly seen in Western populations. The diagnostic features of each of these 2 types of chronic fungal sinusitis ${ }^{(1)}$ are listed in Table 1. Rarely, CGFS may coexist with other types of fungal sinusitis, particularly allergic fungal sinusitis ${ }^{(3)}$. CGFS is most often caused by Aspergillus flavus and hence variably referred to as invasive rhinoorbital or rhinocerebral aspergillosis ${ }^{(1)}$.

The selection of an appropriate antifungal drug for treatment of invasive fungal sinusitis is largely based on clinical reports of patient's response to therapy rather than on antifungal susceptibility testing, which has limited reliability at present ${ }^{(4)}$. For several years, the drug of choice for all invasive fungal infections was amphotericin B. The drug binds to ergosterol of the fungal cell 
Table 1. Diagnostic features of chronic granulomatous fungal sinusitis and chronic invasive fungal sinusitis.

Chronic granulomatous fungal sinusitis

Geographical Chiefly Sudan, India, Pakistan and Saudi Arabia prevalence

$\begin{array}{lll}\text { Clinical } & - & \text { Mostly in immunocompetent patients } \\ \text { features } & \quad & \text { May be diabetic } \\ & \quad & \text { Gradual onset } \\ & \quad \text { Indolent course } \\ & \quad \text { Unilateral proptosis, cheek or orbital swelling common } \\ & \text { Recurrences common }\end{array}$

Histological - Regional tissue invasion by fungal hyphae which are scanty . features - Non caseating granulomas with foreign body or Langhan's type giant cells which may contain hyphae along with lymphocytes and plasma cells

- Occasional presence of central microgranulomata of eosinophils, fibrinoid necrosis, perivascular fibrosis and vasculitis

- Considerable fibrosis may be present

\section{Chronic invasive fungal sinusitis}

Chiefly described in Western populations

- Mostly in immunodeficient patients

- May be diabetic

- Gradual onset

- Progressive course

- Usually commences in deeper sinuses like posterior ethmoid and sphenoid

Tissue necrosis with sparse chronic inflammatory infiltrate Numerous fungal hyphae see

Invasion of mucosa, submucosa, blood vessels and bone by fungal hyphae membrane and makes it porous, leading to leakage of intracellular ions and cell death. The drug is associated with significant side-effects like nephrotoxicity, thrombophlebitis, fever and chills and cardiac arrhythmia. Lipid formulations of the drug such as lipid soluble amphotericin B and liposomal amphotericin $B$ have less toxicity but are expensive.

The azole derivatives, introduced in the late 1980s, act by selectively inhibiting fungal cytochrome P450 enzymes, which are involved in ergosterol synthesis, an early step in fungal cell membrane formation. Azoles can be classified into imidazoles or triazoles. The latter are characterized by three nitrogen atoms in the five membered azole ring and include itraconazole, fluconazole, voriconazole and posaconazole. Itraconazole has been shown to be effective in treating CGFS, often in combination with amphotericin $\mathrm{B}^{(5-8)}$. While in some cases the drug was administered in a planned manner before or after a course of amphotericin $B^{(5,8)}$, in other cases, the drug was given when the patient became intolerant to amphotericin $B^{(7)}$ or showed persistent disease ${ }^{(6)}$. Voriconazole is a triazole drug, which has shown great efficacy in patients with invasive aspergillosis ${ }^{(9,10)}$. The drug is well absorbed orally and has few side-effects, making it a very attractive alternative to amphotericin B. Currently, it is recommended as a first line drug for treatment of invasive aspergillosis by the Infectious Disease Society of America (11).

In the present study, we aimed to review the outcome following therapy with different kinds of antifungals in a series of patients with CGFS to develop an effective protocol for antifungal therapy for future use in such patients.

\section{Materials and methods}

We reviewed the clinical records of patients admitted with a diagnosis of CGFS in the ENT department of our hospital between January 2006 to May 2014 to note the clinical presentation, fungal culture results, histopathological features, radiological findings, surgical procedures, antifungal agents administered and outcome following treatment. We included only patients with biopsy proven evidence of CGFS who also had a positive smear and/or fungal culture. All patients were followed up at intervals of 1 to 3 months after commencing therapy. The clinical findings, rigid nasal endoscopy findings and radiological findings (whenever possible) were noted at each follow-up. The duration of follow-up for each patient was calculated from the date of initial presentation.

\section{Statistical analysis}

Descriptive statistics and frequency and percentages were reported for categorical variables. Fisher's exact test (2 tailed) was done to compare outcomes following first line therapy and to compare outcomes in relation to preoperative staging of the disease.

\section{Institutional Review Board approval}

Approval of the Institutional Review Board and Ethics committee, Christian Medical College, Vellore, was obtained for the performance of this study (IRB no. 8971).

\section{Results}

\section{Demography}

The study included 14 males and 4 females whose ages ranged from 20 to 65 years ( mean $=46.4$ years). 


\section{Symptoms}

The duration of symptoms varied from 1 month to 7 years with a mean of 22.9 months. The most common presenting symptoms were visual deterioration (44.4\%), proptosis (38.9\%), facial pain (38.9\%), headache (33.3\%) and nasal obstruction (33.3\%). The disease was unilateral in all except 3 patients in whom the disease started as a unilateral lesion that progressed to become bilateral. Of 8 patients (44.4\%) who had visual deterioration, 3 had total loss of vision in one eye.

\section{Signs}

Six (33.3\%) patients had periorbital swelling and 5 (27.8\%) had ipsilateral cheek swelling. On rigid nasal endoscopic examination, only 4 patients had an obvious nasal mass which was amenable to biopsy. Three (16.7\%) patients had sinonasal polyposis.

\section{Prior surgery}

Nine (50\%) patients had undergone at least 1 surgical procedure involving the sinuses prior to presentation. These included endoscopic sinus surgery in 7 patients, Caldwell Luc surgery in 2 patients, lateral rhinotomy and excision sinonasal mass in one and dental extraction followed by transalveolar biopsy and curettage in another.

\section{Comorbidities}

Six (33.3\%) patients had diabetes mellitus and 4 (22.2\%) were hypertensive. One patient had chronic renal failure.

\section{Fungal smear and culture results}

A. flavus was identified in 16 (88.9\%) patients. Three patients (16.7\%) had Mucor species, 1 with a combination with A. flavus and Mucor species, another with a combination of A. flavus and Rhizopus species and yet another with $M$. circinelloides alone. Fungal culture was negative in 1 patient.

\section{Radiological findings}

Based on the radiological extent, patients were categorized into 3 groups (Table 2). Stage $1(n=4)$ consisted of patients with completely resectable disease confined to the nose and paranasal sinuses. Stage $2(n=8)$ consisted of patients with completely resectable disease with extension to adjacent areas like the orbit and palate. Stage $3(n=6)$ consisted of patients with partially resectable disease or intracranial extension. Involvement of an individual sinus was best seen in contrast enhanced CT scan of the nose and paranasal sinuses, which showed soft tissue thickening with mild enhancement. Intracranial extension and dural involvement was best assessed by contrast enhanced MRI scanning of the paranasal sinuses and brain.

\section{Histopathology results}

All patients had features of CGFS. Typically, the tissue showed a
Table 2. Staging of disease.

\begin{tabular}{|c|c|c|}
\hline & Areas involved & Surgery \\
\hline Stage 1 & $\begin{array}{l}\text { Nose \& paranasal sinuses } \\
\text { alone }\end{array}$ & $\begin{array}{l}\text { Endoscopic resection; } \\
\text { for maxilla partial / total } \\
\text { maxillectomy }\end{array}$ \\
\hline Stage 2 & $\begin{array}{l}\text { Paranasal sinuses with } \\
\text { immediate adjacent areas } \\
\text { which are surgically resec- } \\
\text { table with minimal morbi- } \\
\text { dity eg. orbit (extraconal), } \\
\text { palate \& oral cavity }\end{array}$ & $\begin{array}{l}\text { Endoscopic / open resec- } \\
\text { tion; for maxilla partial / } \\
\text { total maxillectomy }\end{array}$ \\
\hline Stage 3 & $\begin{array}{l}\text { Intracranial extension } \\
\text { (extradural/ intracerebral) } \\
\text { or partially resectable with } \\
\text { extension to pterygopala- } \\
\text { tine fossa, cavernous sinus, } \\
\text { cheek and periorbital } \\
\text { region }\end{array}$ & $\begin{array}{l}\text { Combined endoscopic } \\
\text { and open approach with } \\
\text { craniofacial resection } \\
\text { for anterior skull base / } \\
\text { frontal lobe disease }\end{array}$ \\
\hline
\end{tabular}

chronic infiltrate of lymphocytes, plasma cells, histiocytes and Langhans type multinucleate giant cells with epithelioid granuloma with or without necrosis. Fungal hyphae were invariably seen invading tissue or bone. Angioinvasion was not seen in any specimen.

Four of the patients in this series were diagnosed as having mixed fungal sinusitis with a combination of CGFS and allergic fungal sinusitis. These cases have been reported before ${ }^{(3)}$. All these patients had predominant features of CGFS and areas with allergic fungal sinusitis.

\section{Surgical procedures}

Most patients (82.4\%) underwent endoscopic excision of the sinonasal mass; in 2 patients this was combined with Caldwell Luc surgery and in another 2 with orbital exenteration. Both patients who had undergone orbital exenteration had total loss of vision in the affected eye. One patient underwent total maxillectomy and another underwent craniofacial resection of a frontal lobe mass. Operative findings most often included the finding of a pink or pale, firm, gritty mass with little vascularity infiltrating the underlying tissue. In patients with partial visual loss, orbital debridement was performed to the extent possible.

\section{Antifungal agents (Table 3)}

\section{Amphotericin $B$}

Intravenous amphotericin B was commenced as first line of therapy after surgery in 6 patients (38.9\%), 3 of whom had stage 2 disease and 3 of whom had stage 3 disease. Plain amphotericin $B$ was administered to 4 patients and liposomal amphotericin $B$ to another 2 patients. Three patients (50\%) were intolerant to amphotericin B, one even with liposomal amphotericin B. These patients discontinued the drug without taking the full dose and 
Table 3. Response to first line therapy in relation to type of drug used and stage of disease $(n=18)$.

\begin{tabular}{|c|c|c|c|c|c|c|c|c|c|c|c|c|}
\hline \multirow[b]{3}{*}{ Stage of disease } & \multicolumn{12}{|c|}{ Drugs given as first line therapy } \\
\hline & \multicolumn{3}{|c|}{$\begin{array}{l}\text { Amphotericin B } \\
\text { alone }(n=5)\end{array}$} & \multicolumn{3}{|c|}{$\begin{array}{l}\text { Amphotericin } B+ \\
\text { Itraconazole }(n=1)\end{array}$} & \multicolumn{3}{|c|}{$\begin{array}{l}\text { Itraconazole } \\
\text { alone }(n=8)\end{array}$} & \multicolumn{3}{|c|}{$\begin{array}{l}\text { Voriconazole } \\
\text { alone }(n=4)\end{array}$} \\
\hline & 1 & 2 & 3 & 1 & 2 & 3 & 1 & 2 & 3 & 1 & 2 & 3 \\
\hline Residual disease $(n=5)$ & & 3 & 2 & & & & & & & & & \\
\hline No evidence of disease $(n=13)$ & & & & & & 1 & 3 & 4 & $1^{*}$ & 1 & 1 & 2 \\
\hline
\end{tabular}

had residual disease.

All except 1 of 6 patients who received amphotericin B as first line therapy had residual disease at the end of therapy. The sole patient without residual disease had received a combination of liposomal amphotericin B and itraconazole.

\section{Itraconazole}

Nine (50\%) patients received itraconazole as first line therapy. Of these, 3 had stage 1 disease, 4 had stage 2 disease and 2 had stage 3 disease. All (except 1 patient) took the drug for a minimum of 6 months. One patient with stage 3 disease received the drug along with amphotericin B as first line therapy. Eight patients were disease-free at follow-up, which ranged from 6 months to 10 years. The sole patient with stage 3 disease who had taken the drug for 3 months had a poor general condition and died of unrelated causes.

Three (16.6\%) other patients with residual disease after failure / intolerance to amphotericin B were disease free after treatment with itraconazole for 1 year.

\section{Voriconazole}

Four patients (22\%) were given oral voriconazole at a dose of $200 \mathrm{mg}$ twice daily as a first line of therapy. Of these 1 had stage 1 disease, another had stage 2 disease, and the other 2 had stage 3 disease. Two more patients who had residual disease following amphotericin $B$ therapy received voriconazole as second line therapy for 1 year. Both were disease free at last follow-up.

\section{Follow-up (Table 3)}

Overall follow-up ranged from 6 months to 8 years with a mean of 2.6 years. Of a total of 18 patients, one patient who died from intercurrent illness was excluded from the analysis. All 12 patients who received one of the azoles alone as first line therapy showed no evidence of disease at follow-up, while all 5 patients who received amphotericin $B$ alone as first line therapy had residual disease at follow-up and the difference between the groups was statistically significant $(p=0.001)$.

Assessment of disease status was based on clinical and endoscopic resolution of disease in all patients. Post-treatment contrast enhanced CT scans were available in all those with residual disease $(27.8 \%)$ and 4 (22.2\%) of those with no evidence of disease. All 5 with residual disease and 7 patients with no disease also had biopsy of sinonasal lesions or mucosa confirming the presence / absence of residual disease. One patient died during the course of treatment due to unrelated causes. Four patients who are still on therapy have been disease free clinically and endoscopically over the last 6-9 months of treatment.

When comparing the overall therapeutic response to first line therapy based on pre-treatment staging of disease, there was no significant difference between those patients who had completely resectable disease (stages 1 and 2 ) and those with partially resectable disease/ intracranial extension (stage 3$)(p=0.6)$

\section{Discussion}

The therapy of CGFS has been controversial and there are no clearcut guidelines on staging and therapy. The disease occurs chiefly in Sudan and Asia and there are a limited number of reports concerning treatment and follow-up from this region.

The various treatment options that are reported in the literature are considered below.

\section{First line Single agent therapy}

\section{Amphotericin B}

For several years, the gold standard of therapy for all types of invasive fungal sinusitis in both immunocompromised and immunocompetent patients has been amphotericin B. The optimal dose of intravenous amphotericin B is controversial. A dose of 3-5 gm is ideal but few patients are able to tolerate such high doses, because of its toxicity. There are frequent reports of stopping the drug within a few days of commencement of therapy ${ }^{(8,12)}$. In the present series, 4 patients had to discontinue the drug because of toxicity. The efficacy of amphotericin B against aspergillus is variable ${ }^{(13,14)}$. In our series only one of the 6 patients who received amphotericin $B$ alone had no residual disease on follow-up. This patient had also received oral itraconazole concurrently. 
Protocol for management of CGFS

\section{Itraconazole}

Itraconazole is an azole derivative, which selectively inhibits fungal cytochrome P450 enzymes and reduces the synthesis of ergosterol, a component of the fungal cell membrane. The drug is well absorbed orally after a meal but has poor CSF penetration. The exact dose and duration of itraconazole therapy is poorly defined. Gumaa et al. ${ }^{(15)}$ described good response to oral itraconazole at a dose of $100 \mathrm{mg}$ twice daily for 12-19 months in 22 patients. Higher doses of $200 \mathrm{mg}$ twice daily have also been recommended. Reports of good response to itraconazole, with or without amphotericin B, have been described by others too ${ }^{(8)}$. Seven of the 8 patients in our series who had received itraconazole alone with long-term follow-up also showed a good response.

\section{Voriconazole}

The sole randomized, controlled trial of voriconazole (given at a dose of $6 \mathrm{mg} / \mathrm{Kg}$ IV twice daily for 1 day followed by $4 \mathrm{mg} /$ $\mathrm{Kg}$ twice daily) versus plain amphotericin $\mathrm{B}$ (given at a dose of $1-1.5 \mathrm{mg} / \mathrm{Kg} /$ Day) in 277 immunocompromised patients with aspergillosis showed greater antifungal efficacy and improved survival at 12 weeks for voriconazole ${ }^{(9)}$. Based on this study, the current guidelines of the Infectious Disease Society of America are that voriconazole should be given as first line therapy for invasive aspergillosis of the sinuses.

There are fewer examples of therapy with voriconazole for CGFS in immunocompetent patients. Nakaya et al. ${ }^{(10)}$ describe 3 patients with sinonasal CGFS who responded successfully to oral voriconazole. Our experience with 6 patients who received postoperative voriconazole, 3 as first line therapy and 3 as second line therapy following failure of response to amphotericin $B$ has been encouraging. The 3 patients who received the drug as first line therapy had advanced disease with intracranial extension and yet responded favourably. The advantage of voriconazole over amphotericin $B$ is that it is effective when given orally and the direct and indirect costs of administering the drug are substantially less as no hospitalisation is required. However, it is more expensive than itraconazole.

The optimal duration of therapy of oral antifungals is controversial, with reports ranging from 6 to 18 months ${ }^{(5,10)}$. The risk of recurrence necessitates prolonged treatment. In the present series, most patients with advanced disease received oral voriconazole for at least 9 months to 1 year with minimal side effects. Transient rise in liver enzymes not requiring stoppage of therapy, was seen in 2 patients.

II. First line combination therapy: amphotericin B with itraconazole

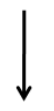

Diagnosis by fungal smear and histopathology+/- fungal culture

Staging of disease based on clinical and radiological extent (see Table 2)

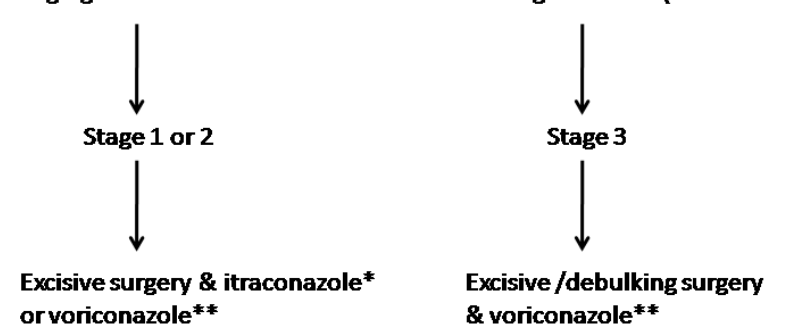

*Oral itraconazole at a dose of $200 \mathrm{mg}$ twice daily for 9-12 months ** Oral voriconazole at a dose of $200 \mathrm{mg}$ twice daily for 9-12 months

Figure 1. Suggested protocol for management of chronic granulomatous fungal sinusitis.

\section{Simultaneous therapy}

In a series of 21 patients with invasive aspergillosis, half of whom were given a combination of amphotericin $B$ and itraconazole (16), the authors found that the response to combination therapy was much better (82\%) than single drug therapy with amphotericin $\mathrm{B}$ alone (50\%). No patient was given itraconazole alone in this series. Combination therapy was also found to be useful by other authors ${ }^{(5,7)}$.

In the present series, we had only one patient with stage 3 disease who received a combination of itraconazole and amphotericin B. This patient did not show recurrent disease at follow-up of 1 year and 3 months. The efficacy of this regimen could be attributed to the effect of itraconazole in this case, as all the other 5 patients who received amphotericin $B$ alone had residual disease.

\section{Sequential therapy}

In a report of 11 patients with craniocerebral aspergillosis who were treated with sequential intravenous amphotericin B and itraconazole ${ }^{(8)}, 5$ patients failed to receive the full dose of amphotericin $B$ because they developed intolerance to the drug. These patients received a prolonged course of itraconazole (for 8 to 12 months or longer) and showed a good response, suggesting that itraconazole seemed to have effected the cure. Similar findings were noted by others ${ }^{(12)}$.

\section{Salvage therapy}

Azoles have been used as salvage rather than as first line therapy in many reports ${ }^{(6,7)}$. The advantage of azoles is that they can be given orally and have few side-effects. Hence, they are often used for long-term therapy to prevent recurrence. 


\section{No medical therapy}

Isolated reports of cases of CGFS who have responded to excisive surgery without antifungal therapy exist in the literature ${ }^{(6,17)}$. In one report, all 5 patients who did not receive postoperative antifungal therapy showed relapse of disease ${ }^{(14)}$. None of our patients had resolution of disease with surgery alone and our view is that administration of systemic antifungal therapy is essential for invasive fungal disease.

\section{Our experience}

Our experience has been that patients with stage 1 and 2 disease do best with either postoperative itraconazole or voriconazole and those with stage 3 with postoperative voriconazole (Figure 1). Irrespective of the staging, all patients in the present series who were given amphotericin $B$ alone showed evidence of residual or recurrent disease, making this drug unattractive as first/second line therapy for CGFS. Response to therapy is clearly more related to the type of antifungal drug used than the stage at presentation, if excisive surgery has been performed adequately.

\section{Author contributions}

VR: Study concept and design, acquisition of data, analysis of data, interpretation of data, drafting of the manuscript, critical revision of the manuscript. MS: acquisition of data, drafting of the manuscript, critical revision of the manuscript. JAE: acquisition of data, drafting of the manuscript, critical revision of the manuscript. SSM: acquisition of data, drafting of the manuscript, critical revision of the manuscript.

\section{Conflict of interest}

The authors declare that there is no conflict of interest.

\section{References}

1. deShazo RD, O'Brien M, Chapin K, SotoAguilar M, Gardner L, Swain R. A new classification and diagnostic criteria for invasive fungal sinusitis. Arch Otolaryngol Head Neck Surg. 1997; 123: 1181-1188.

2. Veress B, Malik OA, el-Tayeb AA, el-Daoud S, Mahgoub ES, el-Hassan AM. Further observations on primary paranasal Aspergillus granuloma in the Sudan. A morphologica study of 46 cases. Am J Trop Med Hyg. 1973; 2: 765-772.

3. Rupa V, Thomas M. Different types of fungal sinusitis occurring concurrently: implications for therapy. Eur Arch Otorhinolaryngol. 2013; 270: 603-608.

4. Ghannoum MA, Rex JH, Galgiani JN Susceptibility testing of fungi: current status of correlation of in vitro data with clinical outcome. J Clin Microbiol. 1996; 34: 489495.

5. Panda NK, Saravanan K, Chakrabarthi A. Combination antifungal therapy for invasive aspergillosis: can it replace high-risk surgery at the skull base? Am J Otolaryngol. 2008; 29: 24-30.

6. Busaba NY, Colden DG, Faquin WC, Salman SD. Chronic invasive fungal sinusitis: a report of two atypical cases. Ear Nose Throat J. 2002; 81: 462-466.

7. Streppel M, Bachmann G, Damm M, Arnold G, Stennert E.Successful treatment of an invasive aspergillosis of the skull base and paranasal sinuses with liposomal ampho- tericin B and itraconazole. Ann Otol Rhinol Laryngol. 1999; 108: 205-207.

8. Siddiqui AA, Shah AA, Bashir S Craniocerebral aspergillosis of sinonasal originin immunocompetent patients: clinical spectrum and outcome in 25 cases Neurosurgery. 2004; 55: 602-613.

9. Herbrecht R, Denning DW, Patterson TF, et al. Voriconazole versus amphotericin $\mathrm{B}$ for primary therapy of invasive aspergillosis. $\mathrm{N}$ Engl J Med. 2002; 347: 408-415.

10. Nakaya K, Oshima T, Kudo T, et al. New treatment for invasive fungal sinusitis: three cases of chronic invasive fungal sinusitis treated with surgery and voriconazole. Auris Nasus Larynx. 2010; 37: 244-249.

11. Walsh TJ, Anaissie EJ, Denning DW, et al. Treatment of aspergillosis: clinical practice guidelines of the Infectious Diseases Society of America. Clin Infect Dis. 2008; 46: 327-360.

12. Kim TH, Jang HU, Jung $Y Y$, Kim JS Granulomatous invasive fungal rhinosinusitis extending into the pterygopalatine fossa and orbital floor: A case report. Med Mycol Case Rep. 2012; 1: 107-111.

13. Stringer SP, Ryan MW. Chronic invasive fungal rhinosinusitis. Otolaryngol Clin North Am. 2000; 33: 375-387.

14. Alrajhi AA, Enani M, Mahasin Z, Al-Omran Chronic invasive aspergillosis of the paranasal sinuses in immunocompetent hosts from Saudi Arabia. Am J Trop Med Hyg. 2001; 65: 83-86.
15. Gumaa SA, Mahgoub ES, Hay RJ. Postoperative responses of paranasal Aspergillus granuloma to itraconazole. Trans R Soc Trop Med Hyg. 1992; 86: 93-94.

16. Popp Al, White MH, Quadri T, Walshe L, Armstrong D. Amphotericin B with and without itraconazole for invasive aspergillosis. A three year retrospective study. Int J Infect Dis. 1999; 3: 157-160.

17. Thakkar A, Sarkar C, Dhiwakar M, Bahadur S, Dahiya S. Allergic fungal sinusitis: expanding the clinicopathological spectrum Otolaryngol Head Neck Surg. 2004; 130: 209-216.

Dr. V. Rupa, M.S., D.L.O.

Professor and Head

Department of ENT Unit 3

Christian Medical College Hospital

Vellore -632004

India

Tel: $+91-416-2283485$

Fax: +91-416-223 2035

E-mail: rupavedantam@cmcvellore.

ac.in 